\title{
Computerised tomography scan in multi-drug-resistant versus extensively drug-resistant tuberculosis
}

\author{
Payam Mehrian ${ }^{1 A, D}$, Poopak Farnia ${ }^{2,3 C}$, Dina Jalalvand ${ }^{2 E}$, Mostafa Rezaei Chamanii ${ }^{2 B, F}$, Mahmood Bakhtiyari ${ }^{2 C}$ \\ 'Telemedicine Research Center, National Research Institute of Tuberculosis and Lung Diseases (NRITLD), Shahid Beheshti University of Medical Sciences, \\ Tehran, Iran \\ ${ }^{2}$ Mycobacteriology Research Centre (MRC), National Research Institute of Tuberculosis and Lung Disease (NRITLD), Shahid Beheshti University of Medical \\ Sciences, Tehran, Iran \\ ${ }^{3}$ Department of Biotechnology, School of Advanced Technologies in Medicine, Shahid Beheshti University of Medical Sciences, Tehran, Iran
}

\section{Abstract}

Purpose: Multi-drug-resistant tuberculosis (MDR-TB) is simultaneously resistant to isoniazid and rifampin. Of course, this germ may also be resistant to other anti-tuberculosis drugs. Patients with extensively drug-resistant tuberculosis (XDR-TB) are also resistant to all types of fluoroquinolone and at least one of the three injectable medications: amikacin, clarithromycin, or kanamycin, in addition to isoniazid and rifampin. Therefore, the main objective of the current study was to evaluate and compare the computed tomography (CT) scan findings of MDR-TB and XDR-TB patients.

Material and methods: In this comparative descriptive cross-sectional study 45 consecutive TB patients who referred to Masih Daneshvari Hospital, Tehran, Iran from 2013 to 2019 were enrolled. TB was diagnosed based on sputum smear and sensitive molecular and microbial tests. Patients were divided into two groups (MDR-TB and XDR-TB) based on two types of drug resistance. CT scan findings were compared for cavitary, parenchymal, and non-parenchymal disorders. The early diagnostic values of these factors were also calculated.

Results: Findings related to cavitary lesions including the pattern, number, size of the largest cavity, maximum thickness of the cavity, lung involvement, number of lobes involved, and the air-fluid levels in the two patient groups were similar $(p>0.05)$. Parenchymal findings of the lung also included fewer and more nodules of $10 \mathrm{~mm}$ in the MDR-TB and XDR-TB groups, respectively. Tree-in-bud, ground-glass-opacity, bronchiectasis, cicatricial emphysema, and lobar atelectasis were similar in the two patient groups $(p>0.05)$. Findings outside the parenchymal lung, including mediastinal lymphadenopathy and pericardial effusion, showed no statistically significant difference between the MDR-TB and XDR-TB groups $(p>0.05)$. Parenchymal calcification was more common in the XDR group than in the MDR group (64.7\% and $28.6 \%$, respectively) with a significant difference $(p=0.01)$.

Conclusions: CT scan findings in patients with XDR-TB are similar to those of patients with MDR-TB for cavitary, parenchymal, and non-parenchymal lung characteristics. However, patients with XDR-TB tend to have more parenchymal calcification and left-sided plural effusion. CT characteristics overlap between XDR-TB and those with MDR-TB. It can be concluded that CT scan features are not sensitive to the diagnosis.

Key words: tuberculosis, antimicrobial resistance, computerised tomography scan.

\section{Introduction}

Tuberculosis (TB) is an ancient disease occurring worldwide with numerous early and late complications $[1,2]$.
According to the World Health Organization (WHO) definition, multi-drug-resistant tuberculosis (MDR-TB) is resistant to both isoniazid and rifampin [3]. Of course, this germ may also be resistant to other anti-tuberculosis

\footnotetext{
Correspondence address:

Dr. Dina Jalalvand, Shahid Beheshti University of Medical Sciences, 1068, block 5, Ekbatan town, Tehran, Iran, 1396843193, Tehran, Iran,

e-mail: Dinajalalvand@gmail.com
}

Authors' contribution:

A Study design · B Data collection · C Statistical analysis · D Data interpretation · E Manuscript preparation · F Literature search · G Funds collection 
drugs [3]. Lack of compliance by patients and self-discontinuation are the main causes for drug resistance as well as single therapy and background diseases [3].

Patients with extensively drug-resistant tuberculosis (XDR-TB) are resistant to all types of fluoroquinolone and at least one of the three injectable medications (amikacin, clarithromycin, or kanamycin) in addition to isoniazid and rifampin [4]. In such cases, two years of continuous therapy is mandatory with direct observation by a physician [4]. In total, $3.2 \%$ of tuberculosis cases worldwide are drug resistant [4]. Usually MDR patients are younger and have multiple treatment sessions and cavitary involvement [5]. Despite multiple studies comparing the clinical findings of MDR and XDR cases [6-8], few studies have compared imaging findings and TB characteristics [7,916]. Therefore, the main objective of the current study was to evaluate and compare the CT scan findings of MDR-TB and XDR-TB patients.

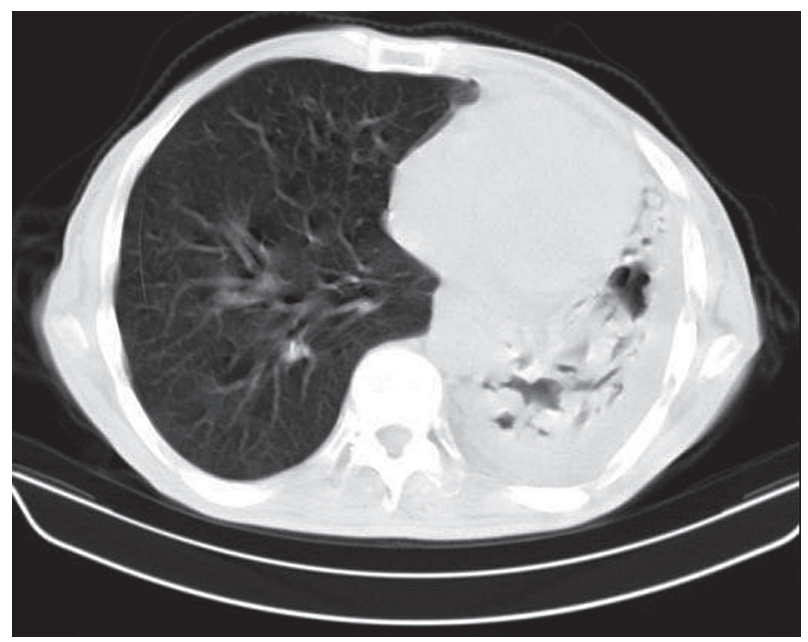

Figure 1.66 year-old male with extensively drug-resistant tuberculosis. Axial computed tomography scan of the lung showing cavitary consolidation with airbronchogram in left lower lobe

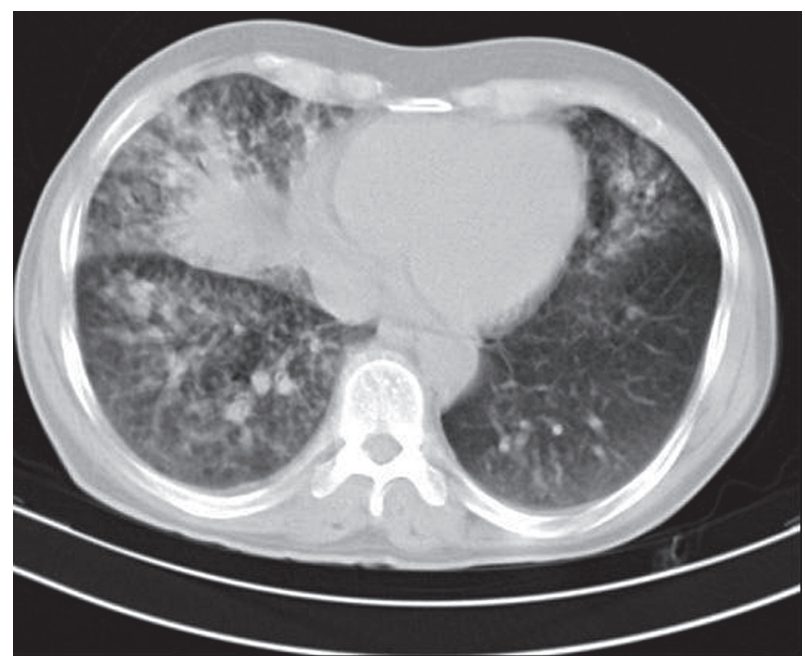

Figure 3.51 year-old male with multi-drug-resistant tuberculosis. Axial computed tomography scan of the lung showing consolidation in right middle lobe with extensive nodular infilteration

\section{Material and methods}

In this comparative descriptive cross-sectional study, a total of 45 consecutive TB patients who referred to Masih Daneshvari Hospital, Tehran, Iran between 2013 and 2019 were enrolled. TB was diagnosed according to the WHO criteria by sputum smear and sensitive molecular and microbial tests. Additional inclusion criteria were age over 18 years, lacking chronic respiratory disease affecting the lung computed tomography (CT) scan findings, and the presence of CT scan results among existing medical documents. Patients were divided into two groups: MDR-TB and XDR-TB, based on two types of drug resistance.

There were 17 eligible cases with XDR-TB and 28 appropriate patients with MDR-TB among $136 \mathrm{MDR}$ cases and 34 patients with XDR. CT scans findings were compared for cavitary, parenchymal, and non-parenchymal

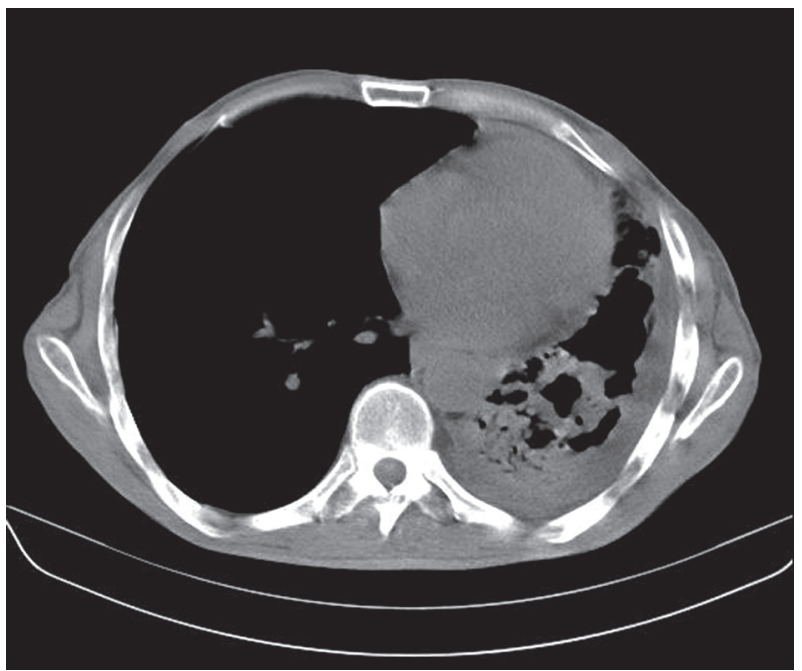

Figure 2. Mediastinal window computed tomography scan of the same patient as in Figure 1 showing left sided pleural effusion

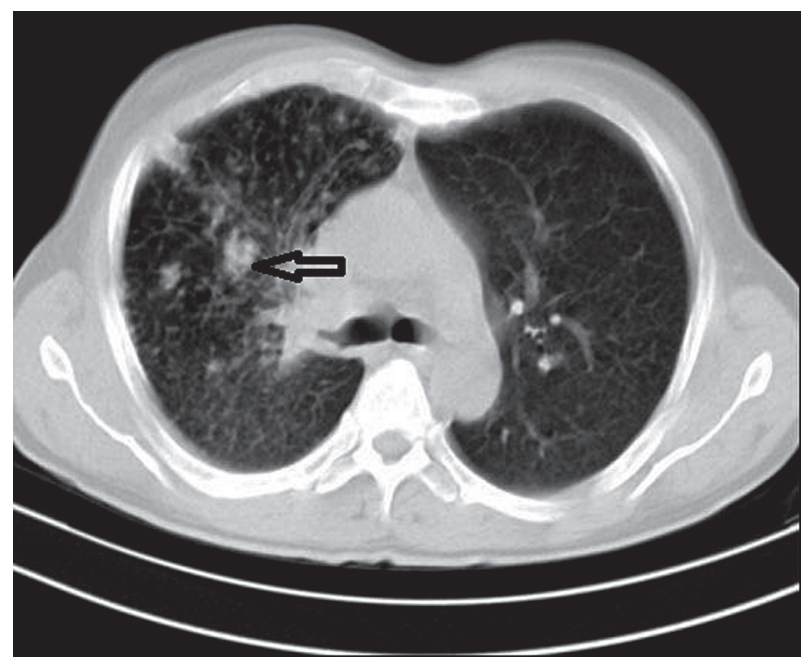

Figure 4. The same patient as in Figure 3. Lung window computed tomography scan showing a macronodule (black arrow) measuring $12 \mathrm{~mm}$ in right upper lobe. Note also micronodular infilteration of the same side 


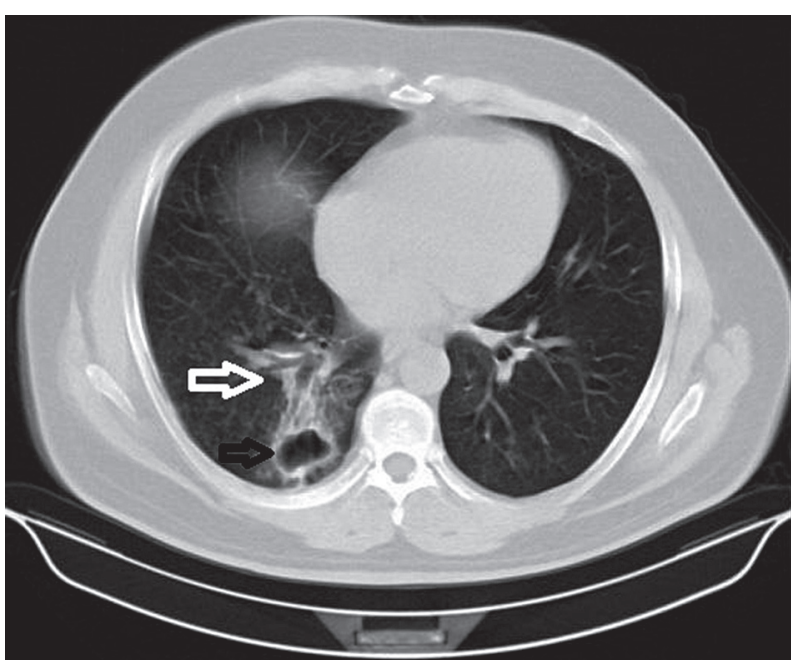

Figure 5. 47 year-old male with extensively drug-resistant tuberculosis. Axial computed tomography scan of the lung showing bronchiectasis (white arrow) with cavity formation (black arrow) in right lower lobe

disorders. The early diagnostic values of these factors were also calculated (Figures 1-6).

Data analysis was done by SPSS version 25.0 software. The categorical variables were shown as frequencies and percentages. The numerical variables were demonstrated as mean and standard deviation. The $\chi^{2}$, KolmogorovSmirnov, independent sample $t$-test, and Mann-Whitney $U$ test were utilised. A $p$-value of less than 0.05 was considered statistically significant.

\section{Results}

The mean patient age was $43.1 \pm 15.2$ years $(44.3 \pm 15.5$ and $41.4 \pm 15.2$ in MDR and XDR cases, respectively; $p>0.05$ ). Among the 45 subjects, 26 were men ( $17 \mathrm{MDR}$ and $9 \mathrm{XDR}$ ) and 19 were women (11 MDR and $8 \mathrm{XDR})$; the difference was not significant $(p>0.05)$. Among the

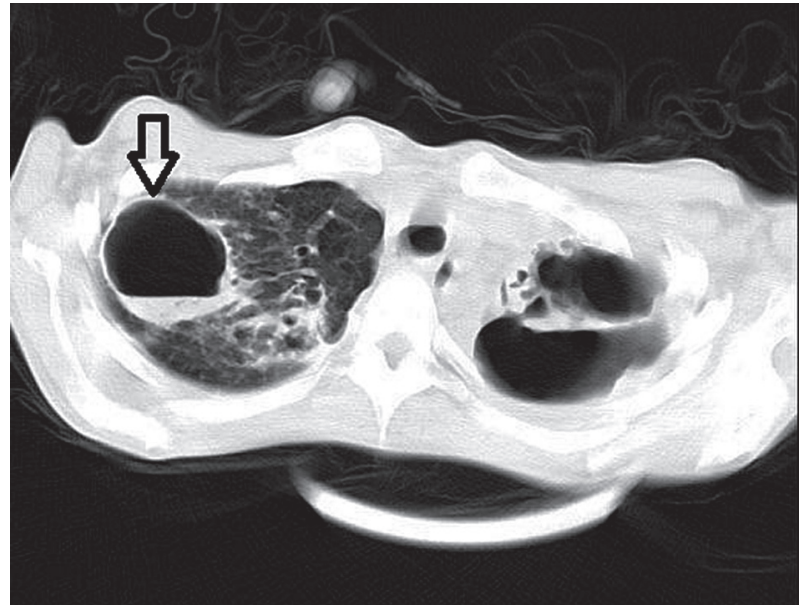

Figure 6. 21 year-old female with extensively drug-resistant tuberculosis. Lung window computed tomography scan showing a cavitary lesion with air-fluid level (black arrow) associated with bronchiectasis in right upper lobe. Note also the fibrocavitary lesion involving left upper lobe

MDR and XDR cases, $21.4 \%$ and $8.9 \%$ were smokers, respectively $(p>0.05)$. Previous first-line anti-TB therapy was positive in $57.8 \%$ and $37.8 \%$ of cases in the MDR and XDR groups, respectively $(p>0.05)$. The mean time intervals between CT scan and positive microbiology results were $26.2 \pm 16$ and $35.5 \pm 14.4$ days, respectively, which showed no significant difference $(p>0.05)$. Diabetes and HIV were seen in seven and six cases, respectively; this difference was not statistically significant $(p>0.05)$.

As shown in Table 1, the findings related to cavitary lesions including the pattern, number, size of the largest, maximum thickness of the cavity, lung involvement, number of involved lobes, and the air-fluid level in the two patient groups were similar $(p>0.05)$. In cavitary lesions, unilateral cases were more common in both MDR and XDR findings.

Table 1. Cavitary lesions across the groups

\begin{tabular}{|l|c|c|c|}
\hline Variable & MDR-TB & XDR-TB & 0.43 \\
\hline Cavitary & $24(85.7 \%)$ & $13(76.5 \%)$ & 0.36 \\
\hline Pattern & \multicolumn{2}{|c|}{} \\
\hline In nodule and mass & $16(57.1 \%)$ & $12(70.6 \%)$ & 0.61 \\
\hline In consolidation & $12(42.9 \%)$ & $6(35.3 \%)$ & 0.21 \\
\hline In nodule and mass plus consolidation & $4(4.13 \%)$ & $5(29.4 \%)$ & 0.39 \\
\hline Total number & $1.5 \pm 1$ & $2 \pm 2$ & 0.44 \\
\hline Size of largest cavitary lesion (mm) & $29 \pm 25$ & $45 \pm 15$ & 0.44 \\
\hline Largest thickness (mm) & $5.5 \pm 2$ & $6 \pm 2$ & 0.12 \\
\hline Laterality & & & 0.25 \\
\hline Unilateral & $29 \pm 25$ & $4(62.9 \%)$ & 0.20 \\
\hline Bilateral & $5.5 \pm 2$ & $1.82 \pm 1.70$ & 0.74 \\
\hline Number of lobes & $1.3 \pm 0.98$ & $5(29.4 \%)$ & \\
\hline Air-fluid level & $7(25.0 \%)$ & & \\
\hline
\end{tabular}


Table 2. Parenchymal lesions across the groups

\begin{tabular}{|c|c|c|c|}
\hline Variable & MDR-TB & XDR-TB & $p$ value \\
\hline Micronodule $<10 \mathrm{~mm}^{* * *}$ & $28(100 \%)$ & $17(100 \%)$ & 1.000 \\
\hline Number of lobes with micronodules* & $3.96 \pm 1.64$ & $3.47 \pm 1.74$ & 0.35 \\
\hline Macronodule $<10 \mathrm{~mm}^{* * *}$ & $15(53.6 \%)$ & $8(47.1 \%)$ & 0.67 \\
\hline Number of lobes with macronodules* & $1(0-2)$ & $0(0-1)$ & 0.58 \\
\hline $\mathrm{TIB}^{* * *}$ & $23(82.1 \%)$ & $15(88.2)$ & 0.58 \\
\hline Number of lobes with TIB* & $2.71 \pm 1.95$ & $2(1-2)$ & 0.83 \\
\hline Ground glass opacity and consolidation*** & $18(64.3 \%)$ & $15(88.2 \%)$ & 0.07 \\
\hline Number of lobes with ground glass opacity and consolidation ${ }^{* *}$ & $1(0-2)$ & $2(1-2)$ & 0.10 \\
\hline Bronchiectasis ${ }^{* * *}$ & $23(82.1 \%)$ & $15(88.2 \%)$ & 0.58 \\
\hline Number of lobes with bronchiectasis ${ }^{* *}$ & $1(0-2)$ & $2(1-3)$ & 0.17 \\
\hline Cicatricial emphysema ${ }^{* * *}$ & $4(14.3 \%)$ & $2(11.8 \%)$ & 0.80 \\
\hline Number of lobes with cicatricial emphysema ${ }^{* *}$ & - & - & 0.85 \\
\hline Parenchymal calcification ${ }^{* * *}$ & $8(28.6 \%)$ & $11(64.7 \%)$ & 0.01 \\
\hline Number of lobes with parenchymal calcification* & $0.5 \pm 0.18$ & $0.76 \pm 0.16$ & 0.32 \\
\hline Lobar atelectasis $^{* * *}$ & $4(14.3 \%)$ & $3(17.6 \%)$ & 0.76 \\
\hline Number of lobes with lobar atelectasis ${ }^{* *}$ & - & - & 0.70 \\
\hline
\end{tabular}

${ }^{*}$ Mean $\pm S D$ tested by independent sample $t$-test

${ }^{*}$ Median and IQR tested by Mann-Whitney $U$ test

${ }^{* * *} \chi^{2}$ test

Table 3. Extra-parenchymal lesions across the groups

\begin{tabular}{|l|c|c|c|}
\hline Variable & MDR-TB & XDR-TB & $p$ value \\
\hline Mediastinal lymphadenopathy & $17(60.7 \%)$ & $10(58.8 \%)$ & 0.90 \\
\hline Pericardial effusion & $2(7.1 \%)$ & $3(17.6 \%)$ & 0.27 \\
\hline Hilar lymphadenopathy & $12(43 \%)$ & $10(58.8 \%)$ & 0.29 \\
\hline Hilar lymphadenopathy laterality & & $9(52.9 \%)$ & 0.16 \\
\hline Unilateral & $9(32.1 \%)$ & $1(5.9 \%)$ & 0.58 \\
\hline Bilateral & $3(10.7 \%)$ & $7(41.2 \%)$ & 0.29 \\
\hline Not seen & $16(57.1 \%)$ & $5(29.4 \%)$ & 0.11 \\
\hline Calcified lymphadenopathy & $3(10.7 \%)$ & $16(94.1 \%)$ & 0.06 \\
\hline Pleural effusion & $20(71.7 \%)$ & & 0.051 \\
\hline Pleural effusion laterality & & $14(82.4 \%)$ & 0.58 \\
\hline Unilateral & $15(53.6 \%)$ & $2(11.8 \%)$ & 0.06 \\
\hline Bilateral & $5(17.9 \%)$ & $1(5.9 \%)$ & 0.58 \\
\hline No effusion & $8(28.6 \%)$ & $3(10.7 \%)$ & \\
\hline Loculated pleural effusion & $3(10.7 \%)$ & & \\
\hline
\end{tabular}

As shown in Table 2, the parenchymal findings of the lung also included fewer and more nodules of $10 \mathrm{~mm}$ in the MDR and XDR groups, respectively. Tree-in-bud, ground-glass-opacity (GGO), bronchiectasis, cicatricial emphysema, and lobar atelectasis were similar in the two patient groups $(p>0.05)$. Parenchymal calcification was more common in the XDR group (64.7\% vs. $28.6 \%)$, with a significant difference $(p=0.01)$. Micronodules, TIB, GGO, and bronchiectasis were more common findings in the XDR group, and micronodules, TIB, and bronchiectasis were more common findings in the MDR group.

As shown in Table 3, the findings outside of the parenchymal lung, including mediastinal lymphadenopathy and pericardial effusion, showed no statistically significant difference between the MDR-TB and XDR-TB groups $(p>0.05)$. However, pleural effusion and mediastinal lymphadenopathy were most common findings in both the $\mathrm{XDR}$ and the MDR group. 


\section{Discussion}

Proper prescription of second-line anti-tuberculosis drugs must be ensured to thoroughly treat existing MDR-TB, reduce further transmission, and prevent the emergence of XDR-TB [17]. Interestingly, CT scanning may be used to monitor therapeutic responses after initial treatments for TB cases [18]. In the current study, the CT scan findings of MDR and XDR tuberculosis patients were compared. Only one significant difference that was related to parenchymal calcification with a significantly higher rate among XDR cases was found. Other common but non-significant findings in the XDR cases included greater cavity thickness, bilateral cavities, number of lobes, GGO, pleural effusion, calcified lymphadenopathy, and consolidation. Overall, this study demonstrated that imaging findings are not useful in distinguishing between MDR and XDR tuberculosis.

Contrary to the current results, Cha et al. [10] noted a significant age difference between MDR and XDR groups. A lack of matching with the group-to-group method may explain the differing results. Some differences may relate to the age at which anti-TB treatment was initiated. Bacilli are currently transmitted to younger patients. Other factors include decreased immune response and sedentary lifestyle. Lee et al. [11] reported that cavities are more common in patients with XDR with further lobe involvement than in MDR patients. Wang et al. [9] reported that multiple cavities with thick-walled status are the most promising imaging sign for diagnosing MDR-TB. This issue was found to be true for both MDR and XDR patients in the current study.

Yeom et al. [14] reported extensive lung involvement in XDR and MDR patients compared with non-resistant cases. This result may be explained by the transposition of microorganism-containing fluid to the bronchial tree and further parenchymal involvement. In the current study, the type of cavitary lesion did not differ between XDR and MDR cases. Cha et al. [10] reported that multiplex cavities are more common in both MDR and XDR cases than in treatment-responsive cases. Fishman et al. [15] showed no difference in cavitary pattern between MDR and XDR cases. Other studies have assessed the level of cavitary involvement in AIDS patients [10,12,13]. Cha et al. [10] reported lower cavitation in immunosuppressed patients with a positive HIV status compared with HIV-negative patients with greater immunity. Therefore, as seen in the current study, cavitary lesions are the main pulmonary finding for MDR or XDR without AIDS.

In the current study, the parenchymal findings were similar across the XDR and MDR groups, except for parenchymal calcification, which had a higher rate in XDR cases than in MDR patients. Furthermore, GGO and consolidation showed a borderline significance of 0.06 . Additional studies with larger sample sizes would demonstrate definitively the relationship status. Cha et al. [10] showed that bronchial obstruction and large nodules are more common in MDR and XDR cases versus non-resistant cases. Hence, the use of CT scanning to identify parenchymal lesions is recommended. The micronodules were also common in the current study, being found in all patients with either MDR or XDR. Yeom et al. [14] also showed a greater rate of parenchymal findings in patients compared with non-resistant cases.

Similarly, Lee et al. [11] showed that micronodules and bronchiectasis are more common in XDR than in MDR cases. This issue would help to disseminate caseous necrosis and granulomatosis inflammation. This finding also showed the higher invasion rate of XDR compared with MDR cases. When tree-in-bud and consolidation are accompanied by such findings, the presence of antimicrobial resistance is suggested, and further microbiological assessments would be beneficial. However, as shown in the current study, there are no distinguishing CT scan differences between MDR and XDR cases. Some studies have compared MDR and XDR cases with non-resistant cases $[9,13]$. Further cases of macronodules and bronchial obstruction were reported in MDR and XDR patients than in non-resistant patients. This higher rate of parenchymal involvement was also reported by Yeom et al. [14]. These findings indicate that the psychopathological mechanism and course of development for MDR and XDR are the same [10].

The current study had some limitations: 1) existing medical data were used, which led to a small sample size from among those with CT scan results; 2) selection bias may be present because CT scans are usually ordered for patients with more severe symptoms with features of greater invasiveness; 3 ) the use of existing data led to decreased control on the imaging protocols, 4) the smaller sample size in the XDR group compared with the MDR group led to decreased potency of the presented analyses; and 5) previous studies [19,20] compared drug-resistant with drug-susceptible cases, whereas the current study could not include a control group among drug-sensitive cases. Enrolment of a control group would help emphasise the actual role of CT scanning in the treatment monitoring of TB patients.

\section{Conclusions}

Overall, it may be concluded that CT scan findings in patients with XDR-TB are similar to those in patients with MDR-TB when considering cavitary, parenchymal, and non-parenchymal lung characteristics. However, XDR-TB patients tend to have more parenchymal calcification and left-sided plural effusion. CT characteristics overlap between patients with TB (XDR-TB) and those with MDR-TB (MDRTB). It can be concluded that CT scan features are not sufficiently accurate to use in diagnosing grade of resistance.

\section{Conflict of interest}

The authors report no conflict of interest. 


\section{References}

1. Ogbo FA, Ogeleka P, Okoro A, et al. Tuberculosis disease burden and attributable risk factors in Nigeria, 1990-2016. Trop Med Health 2018; 46: 34 .

2. Glaziou P, Floyd K, Raviglione MC. Global epidemiology of tuberculosis. Semin Respir Crit Care Med 2018; 39: 271-285.

3. Organization WH. Extensively drug-resistant tuberculosis (XDRTB): recommendations for prevention and control. Wkly Epidemiol Rec 2006; 81: 430-432.

4. Gandhi NR, Moll A, Sturm AW, Pawinski R, Govender T, Lalloo U, et al. Extensively drug-resistant tuberculosis as a cause of death in patients co-infected with tuberculosis and HIV in a rural area of South Africa. Lancet 2006; 368: 1575-1580.

5. Chung MJ, Lee KS, Koh WJ, Kim TS, Kang EY, Kim SM, et al. Drug-sensitive tuberculosis, multidrug-resistant tuberculosis, and nontuberculous mycobacterial pulmonary disease in nonAIDS adults: comparisons of thin-section CT findings. Eur Radiol 2006; 16: 1934-1941.

6. Kim HR, Hwang SS, Kim HJ, et al. Impact of extensive drug resistance on treatment outcomes in non-HIV-infected patients with multidrug-resistant tuberculosis. Clin Infect Dis 2007; 45: 1290-1295.

7. Raviglione MC, Smith IM. XDR tuberculosis - implications for global public health. N Engl J Med 2007; 356: 656-659.

8. Control CfD, Prevention. Emergence of Mycobacterium tuberculosis with extensive resistance to second-line drugs - worldwide, 2000-2004. MMWR Morbidity and mortality weekly report. 2006; 55: 301.

9. Wáng YXJ, Chung MJ, Skrahin A, Rosenthal A, Gabrielian A, Tartakovsky M. Radiological signs associated with pulmonary multi-drug resistant tuberculosis: an analysis of published evidences. Quant Imaging Med Surg 2018; 8: 161-173.

10. Cha J, Lee HY, Lee KS, Koh WJ, Kwon OJ, Yi CA, et al. Radiological findings of extensively drug resistant pulmonary tuberculosis in
non-AIDS adults: comparisons with findings of multidrug-resistant and drug-sensitive tuberculosis. Korean J Radiol 2009; 10: 207-216.

11. Lee ES, Park CM, Goo JM, Yim JJ, Kim HR, Lee HJ, et al. Computed tomography features of extensively drug-resistant pulmonary tuberculosis in non-HIV-infected patients. J Comput Assist Tomogr 2010; 34: 559-563.

12. Song Y, Park C. Drug-resistant tuberculosis including multidrug resistant and extensively drug resistant tuberculosis: their clinical significance and features. Imaging Characteristics 2011.

13. Cheon K (ed.). Comparison of CT findings between MDR-TB and XDR-TB2016: European Congress of Radiology 2017.

14. Yeom JA, Jeong YJ, Jeon D, et al. Imaging findings of primary multidrug-resistant tuberculosis: a comparison with findings of drug-sensitive tuberculosis. J Comput Assist Tomogr 2009; 33: 956 Y960.

15. Fishman JE, Sais GJ, Schwartz DS, Otten J. Radiographic findings and patterns in multidrug resistant tuberculosis. J Thorac Imaging 1998; 13: 65-71.

16. Geng E, Kreiswirth B, Burzynski J, Schluger NW. Clinical and radiographic correlates of primary and reactivation tuberculosis: a molecular epidemiology study. JAMA 2005; 293: 2740-2745.

17. Prasad R. Multidrug and extensively drug-resistant tuberculosis management: Evidences and controversies. Lung India 2012; 29: 154-159.

18. Kim S, Lee J, Lee J. Changes in chest CT findings of pulmonary tuberculosis after linezolid treatment. Springerplus 2013; 2: 615.

19. Li D, He W, Chen B, Lv P. Primary multidrug-resistant tuberculosis versus drug-sensitive tuberculosis in non-HIV-infected patients: Comparisons of CT findings. PLoS One 2017; 12: e0176354.

20. Icksan AG, Napitupulu MRS, Nawas MA, Nurwidya F. Chest X-ray findings comparison between multi-drug-resistant tuberculosis and drug-sensitive tuberculosis. J Nat Sci Biol Med 2018; 9: 42-46. 